\title{
Application of Dynamic Neural Networks and Fuzzy Algorithms in the Modeling of Drug Release
}

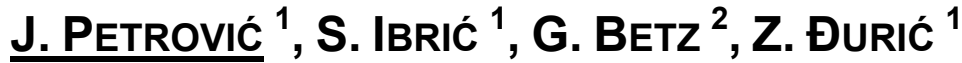 \\ ${ }^{1}$ Institute of Pharmaceutical Technology, Faculty of Pharmacy, University of Belgrade, Belgrade, Serbia \\ ${ }^{2}$ Institute of Pharmaceutical Technology, Pharmacenter, University of Basel, Basel, Switzerland \\ E-mail: jpetrovic@pharmacy.bg.ac.rs (J. Petrović)
}

Sci Pharm. 2010; 78: 709

doi:10.3797/scipharm.cespt.8.PPAT20

The aim of the presented study is to propose the utilization of dynamic neural networks and fuzzy algorithms as in silico tools for prediction of drug release rate from hydrophilic and lipid matrix tablets. Dynamic neural networks are neural networks used for analysis of nonlinear, time dependent processes, having the ability of multiple presentations of inputs to the network. Fuzzy algorithms, on the other hand, enable creation of set of rules defining desired formulation properties. Compositions of matrix tablets were as follows: (a) hydrophilic: polyethylene oxide (Polyox ${ }^{\circledR}$ WSR Coagulant), diclofenac sodium, microcrystalline cellulose (Avicel ${ }^{\circledR} \mathrm{PH} 102$ ); (b) lipid: glyceryl palmitostearate (Precirol ${ }^{\circledR}$ ATO 5), caffeine, mannitol; and magnesium stearate. Matrix tablets, prepared using compression simulator (Zwick 1478), were evaluated on porosity, tensile strength and drug release rate. Commercially available software Peltarion ${ }^{\circledR}$ has been used to construct dynamic neural networks and fuzzy algorithms. Modeling of drug release rate has been conducted taking into account formulation factors and manufacturing method (formulations composition, compression force used for tableting, porosity and tensile strength of manufactured tablets). Focused, Elman's dynamic network was employed to model drug release rate from both hydrophilic and lipid matrix tablets, optimizing the number of neurons in hidden layers as well as signal time delay with genetic algorithms. Separate sets of rules were generated using fuzzy algorithms for hydrophilic and lipid matrix tablets. Testing of the developed dynamic networks and fuzzy algorithms has demonstrated that dissolution profiles for matrix tablets of known composition and mechanical properties can be predicted in silico with great accuracy. In the case of diclofenac sodium release modeling, obtained values for similarity and difference factors for test formulations release profiles were $f_{2}=79,08$ and $f_{1}=4,36$ i. e. $f_{2}=75,23$ and $f_{1}=6,69$. On the other hand, in the case of caffeine release modeling obtained values were $f_{2}=87,61$ and $f_{1}=1,58$ i. e. $f_{2}=72,30$ and $f_{1}=5,24$. For all predicted drug release profiles the correlation coefficient among predicted and true values was higher than 0,9950 . This study demonstrates the feasibility of application of dynamic networks and fuzzy algorithms for modeling of drug release rate from hydrophilic and lipid matrix tablets. 